\title{
Prevalence and new genotypes of Enterocytozoon bieneusi in sheltered dogs and cats in Sichuan province, southwestern China
}

Yalin Zhonga, Ziyao Zhou ${ }^{a}$, Lei Denga ${ }^{a}$ Haifeng Liu, Zhijun Zhong, Xiaoping Ma, Kun Zhang, Yingzhu Wang, Hualin Fu, and Guangneng Peng*

The Key Laboratory of Animal Disease and Human Health of Sichuan Province, College of Veterinary Medicine, Sichuan Agricultural University, Chengdu, Sichuan 611130, PR China

Received 1 February 2021, Accepted 13 March 2021, Published online 2 April 2021

\begin{abstract}
Enterocytozoon bieneusi is a common intracellular parasite that infects a wide range of hosts, including humans and companion animals, raising concerns of zoonotic transmission. However, there is limited epidemiological information on the prevalence and genotypes of E. bieneusi in sheltered dogs and cats in Sichuan province, southwestern China. A total of 880 fecal samples were collected from shelters in different cities of Sichuan province, including 724 samples from dogs, and 156 samples from cats. Enterocytozoon bieneusi was determined by sequence analysis of the ribosomal internal transcribed spacer (ITS). Overall, the prevalence of E. bieneusi was 18\% (158/880), and the parasite was detected in $18.8 \%$ (136/724) and $14.1 \%$ (22/156) of the dogs and cats examined, respectively. Sequence analysis revealed the presence of five genotypes in dogs, including three known genotypes CD9 ( $n=92)$, PtEb IX $(n=41)$, and Type IV $(n=1)$, and two novel genotypes SCD-1 $(n=1)$ and SCD-2 $(n=1)$. Similarly, four genotypes were identified in cats, including CD9 $(n=11)$, Type IV $(n=6), \mathrm{D}(n=4)$, and PtEb IX $(n=1)$. Genotypes D and Type IV have previously been identified in humans and are reported in sheltered dogs and cats in the present study, indicating that these animals could be as potential sources of human microsporidiosis infections.
\end{abstract}

Key words: Enterocytozoon bieneusi, Dogs, Cats, ITS, China, Genotype.

Résumé - Prévalence et nouveaux génotypes d'Enterocytozoon bieneusi chez les chiens et chats de refuges dans la province du Sichuan, dans le sud-ouest de la Chine. Enterocytozoon bieneusi est un parasite intracellulaire commun qui infecte un large éventail d'hôtes, y compris les humains et les animaux de compagnie, ce qui soulève des problèmes de transmission zoonotique. Cependant, il existe peu d'informations épidémiologiques sur la prévalence et les génotypes d'E. bieneusi chez les chiens et les chats des refuges dans la province du Sichuan, au sud-ouest de la Chine. Au total, 880 échantillons de matières fécales ont été prélevés dans des refuges dans différentes villes de la province du Sichuan, dont 724 échantillons de chiens et 156 échantillons de chats. Enterocytozoon bieneusi a été déterminé par analyse de séquence de l'espaceur transcrit interne ribosomique (ITS). Dans l'ensemble, la prévalence d'E. bieneusi était de $18 \%$ (158/880) et le parasite a été détecté chez 18,8\% (136/ $724)$ et $14,1 \%(22 / 156)$ des chiens et des chats examinés, respectivement. L'analyse des séquences a révélé la présence de cinq génotypes chez le chien, dont trois génotypes connus CD9 $(n=92)$, PtEb IX $(n=41)$ et type IV $(n=1)$, et deux nouveaux génotypes SCD-1 $(n=1)$ et $\operatorname{SCD}-2(n=1)$. De même, quatre génotypes ont été identifiés chez les chats, dont CD9 $(n=11)$, Type IV $(n=6), \mathrm{D}(n=4)$ et PtEb IX $(n=1)$. Les génotypes $\mathrm{D}$ et de type IV ont été précédemment identifiés chez l'homme et sont rapportés chez des chiens et des chats des refuges dans la présente étude, ce qui indique que ces animaux pourraient être des sources potentielles d'infections par microsporidiose chez les humains.

\section{Introduction}

Enterocytozoon bieneusi, an obligate intracellular parasite, is the most common microsporidian associated with humans and animals, posing a public health threat $[14,30]$. The disease caused by E. bieneusi is commonly characterized by chronic or severe diarrhea in patients with compromized immune systems, such as acquired immune deficiency syndrome (AIDS) and

*Corresponding author: pgn. si cau@163.com

${ }^{\mathrm{a}}$ These authors contributed equally to this work.

This is an Open Access article distributed under the terms of the Creative Commons Attribution License (https://creativecommons.org/licenses/by/4.0), which permits unrestricted use, distribution, and reproduction in any medium, provided the original work is properly cited. 
organ transplantation recipients [5, 30]. However, some individuals infected with $E$. bieneusi do not exhibit any clinical symptoms, so they can represent carriers of this parasite and spread the spores to susceptible humans and animals [34]. Thus, the National Institute of Allergy and Infectious Diseases (NIAID) has listed E. bieneusi as a Category B Priority Pathogen (https://www.niaid.nih.gov/research/emerging-infectious-diseasespathogens).

Enterocytozoon bieneusi isolates are usually characterized genetically by analysis of the internal transcribed spacer (ITS) region of the ribosomal RNA (rRNA) gene [31, 35]. To date, more than 500 distinct genotypes have been reported in humans and various animals [15]. Phylogenetic analysis has shown that all valid $E$. bieneusi ITS genotypes are clustered into 11 groups [14]. The genotypes in group 1, the largest group, have zoonotic potential, and group 2 is the second largest group and is mainly found in ruminants [14, 22]. The remaining groups, group 3 to group 11, contain genotypes that seem to be hostspecific [7, 14].

Since E. bieneusi was first reported in a Haitian AIDS patient with severe diarrhea [4], and it has been identified in a broad range of wild and domestic animal hosts worldwide, including mammals (e.g. artiodactyls, carnivores, lagomorphs, perissodactyls, primates and rodents) and birds [3, 12, 25, 28, 36]. However, epidemiological data regarding the prevalence and genotypes of E. bieneusi in sheltered dogs and cats in Sichuan province are scarce. Stray dogs and cats infected with E. bieneusi could excrete the infectious spores, which increases the possibility of transmission between animals and humans, thereby posing a potential threat to public health. The purpose of this study was to determine the prevalence and genotypes of E. bieneusi in sheltered dogs and cats. Moreover, the present study also aimed to provide fundamental information for monitoring the transmission of microsporidiosis between humans and stray animals.

\section{Materials and methods}

\section{Ethics statement}

The present study protocol was reviewed and approved by the Research Ethics Committee and the Animal Ethics Committee of Sichuan Agricultural University. Permission was obtained from the shelter's managers before the fecal specimens were collected.

\section{Collection of specimens}

During the period from September 2019 to June 2020, a total of 880 fecal specimens were collected from sheltered dogs and cats, including 724 samples from dogs, and 156 samples from cats. The sampling sites were distributed in different cities in Sichuan Province (Fig. 1). The stray dogs (around 8-12 dogs) were housed in the shared enclosure, so we only collected 3-4 fecal samples for each enclosure. The cats were kept in separate cages, and only one fecal sample was collected from each cat. Fecal samples were collected from the ground or bottom of cages using sterile gloves and then immediately placed into individual $15-\mathrm{mL}$ sterile tubes with ice.

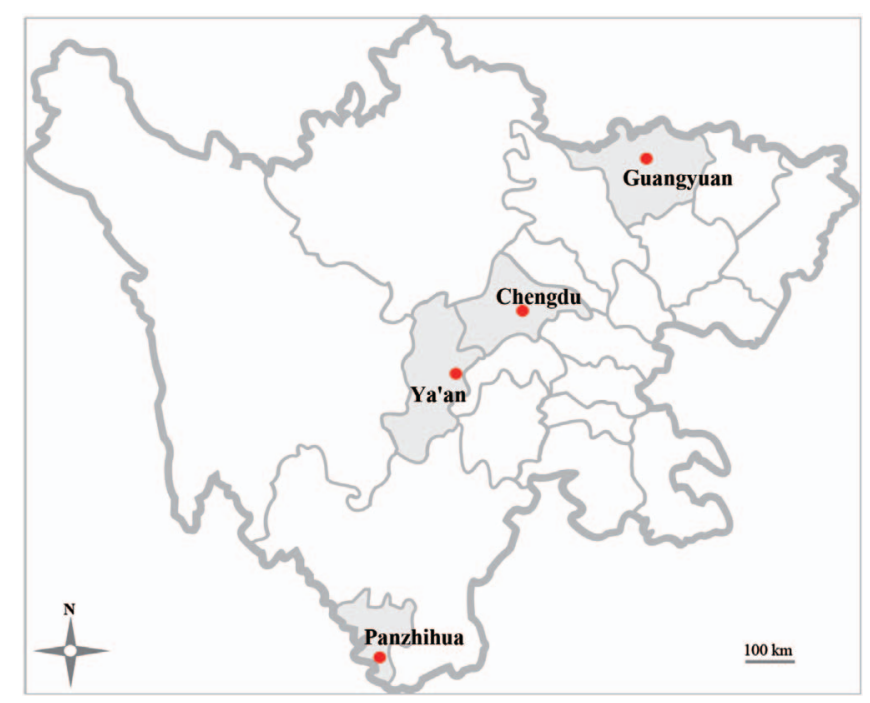

Figure 1. Geographical distribution of the sampled cities (filled red dot) in Sichuan Province.

\section{DNA extraction and PCR amplification}

Genomic DNA was extracted from each fecal sample (around $200 \mathrm{mg}$ ) using the E.Z.N.A.R ${ }^{\circledR}$ Stool DNA kit (Omega Bio-tek Inc., Norcross, GA, USA), according to the manufacturer's instructions. Enterocytozoon bieneusi was determined by nested PCR amplifying the ITS region of the rRNA gene using specific primers as described by Sulaiman et al. [27]. The PCR reaction volume $(25 \mu \mathrm{L})$ contained $12.5 \mu \mathrm{L}$ Taq PCR Master Mix (Sangon Biotech Co., Ltd., Shanghai, China), $1 \mu \mathrm{L}$ each primer $(0.4 \mu \mathrm{M}), 2 \mu \mathrm{L}$ genomic DNA, $1.5 \mathrm{mM}$ $\mathrm{MgCl}_{2}$, and nuclease-free water to volume. Positive and negative controls were included in all the PCR reactions. The secondary PCR products were subjected to $1.5 \%$ agarose gel electrophoresis (AddGene, Watertown, MA, USA) and visualized by staining with SYBR Safe DNA Gel Stain (Thermo Fisher Scientific, Waltham, MA, USA).

\section{Nucleotide sequencing and data analysis}

All secondary PCR products with the expected size (around $392 \mathrm{bp}$ ) were sequenced with primers used in the secondary PCR on an ABI 3730 XL DNA Analyzer. Nucleotide sequences obtained in the present study were aligned with each other and reference sequences downloaded from the GenBank database by using the program Clustal X 1.83 (http://www. clustal.org/). Genotypes of E. bieneusi isolates were grouped by phylogenetic analyses. All genotypes were identified based on $243 \mathrm{bp}$ of the ITS region of E. bieneusi according to the established nomenclature system.

\section{Phylogenetic analysis}

A neighbor-joining tree was constructed to assess the genetic relationships among the E. bieneusi genotypes acquired in this study and those published in previous studies, using the software Mega 6 (http://www.megasoftware.net/), and the 
Table 1. Prevalence and genotypes of E. bieneusi in sheltered dogs from different cities and sources in Sichuan province, southwestern China.

\begin{tabular}{|c|c|c|c|c|c|c|c|}
\hline City & Source & No. examined & No. positive & $\begin{array}{l}\text { Prevalence }(\%) \\
\quad(95 \% \mathrm{CI})\end{array}$ & OR $(95 \% \mathrm{CI})$ & $p$-value & Genotypes $(n)$ \\
\hline \multirow[t]{2}{*}{ Chengdu } & Shuangliu & 158 & 16 & $10.1 \%(5.4-14.8)$ & Reference & & CD9 (8); PtEb IX (7); SCD-1 (1) \\
\hline & Wenjiang & 250 & 80 & $32.0 \%(26.2-37.8)$ & $\begin{array}{c}4.176 \\
(2.336-7.468)\end{array}$ & 0.000 & CD9 (79); PtEb IX (1) \\
\hline Ya'an & Yucheng & 228 & 36 & $15.8 \%(11.1-20.5)$ & $\begin{array}{c}1.664 \\
(0.888-3.117)\end{array}$ & 0.112 & CD9 (4); PtEb IX (32) \\
\hline Panzhihua & Dongqu & 44 & 2 & $4.5 \%(-1.6-10.7)$ & $\begin{array}{c}0.423 \\
(0.093-1.913)\end{array}$ & 0.264 & CD9 (1); PtEb IX (1) \\
\hline Guangyuan & Lizhou & 44 & 2 & $4.5 \%(-1.6-10.7)$ & $\begin{array}{c}0.423 \\
(0.093-1.913)\end{array}$ & 0.264 & Type IV (1); SCD-2 (1) \\
\hline Total & & 724 & 136 & $18.8 \%(15.9-21.6)$ & & & $\begin{array}{c}\text { CD9 (92); PtEb IX (41); } \\
\text { Type IV (1); SCD-1 (1); SCD-2 (1) }\end{array}$ \\
\hline
\end{tabular}

Table 2. Prevalence and genotypes of E. bieneusi in sheltered cats from different cities and sources in Sichuan province, southwestern China.

\begin{tabular}{lccccccc}
\hline City & Source & No. examined & No. positive & $\begin{array}{c}\text { Prevalence } \\
(\%)(95 \% \mathrm{CI})\end{array}$ & OR (95\% CI) & $p$-value & Genotypes $(n)$ \\
\hline Chengdu & Shuangliu & 85 & 13 & $15.3 \%(7.6-22.9)$ & Reference & CD9 (10); D (2); PtEb IX (1) \\
Ya'an & Yucheng & 23 & 3 & $13.0 \%(-0.7-26.8)$ & $0.831(0.215-3.203)$ & 0.788 & CD9 (1); D (2) \\
Panzhihua & Dongqu & 48 & 6 & $12.5 \%(3.1-21.9)$ & $0.791(0.280-2.237)$ & 0.791 & Type IV (6) \\
Total & & 156 & 22 & $14.1 \%(8.6-19.6)$ & & CD9 (11); Type IV (6); \\
& & & & & & D (4); PtEb IX (1) \\
\hline
\end{tabular}

evolutionary distances were calculated using the Kimura twoparameter model. The reliability of these trees was assessed by bootstrap analysis with 1000 replicates. The nucleotide sequences generated in the present study have been deposited in GenBank (https://www.ncbi.nlm.nih.gov/) under accession numbers MW464622 - MW464626 and MW464618 MW464621 for dogs and cats, respectively.

\section{Statistical analysis}

Data were analyzed using SPSS statistical software, version 22 (https://www.ibm.com/analytics/spss-statistics-software) and a Chi-square test was used to detect significant differences. A $p$-value $<0.05$ was considered statistically significant. The adjusted odds ratio (OR) and 95\% confidence interval (CI) for each variable were also calculated.

\section{Results \\ Prevalence of $E$. bieneusi in sheltered dogs and cats}

Overall, the prevalence of E. bieneusi was $18 \%$ (158/880), and the parasite was detected in $18.8 \%$ (136/724) and $14.1 \%$ $(22 / 156)$ of the dogs and cats examined, respectively. In terms of the stray dogs, the prevalence of E. bieneusi among different locations ranged from $4.6 \%$ in Panzhihua and Guangyuan to $32 \%$ in Wenjiang (Table 1). However, the prevalences among different sources were not significantly different statistically $(p>0.05)$. As for stray cats, all tested areas found E. bieneusi-positive samples, with the highest prevalence in Shuangliu $(15.3 \%, 13 / 85)$ (Table 2). The prevalence of E. bieneusi in Yaan and Panzhihua displayed consistent results, with $13 \%$ and $12.5 \%$, respectively. Similarly, there was no statistically significant difference between different sources $(p>0.05)$.

\section{Genetic characterizations of $E$. bieneusi}

A total of five genotypes were identified in stray dogs in the present study by sequence analysis of the nucleotide sequences of the ITS region of E. bieneusi, including three known genotypes (CD9, PtEb IX, and Type IV) and two novel genotypes named SCD-1 and SCD-2 (Table 1). Genotype CD9 was the most prevalent $(92 / 136,67.6 \%)$ and was observed in samples from all the three cities except for Guangyuan, followed by PtEb IX (30.1\%, 41/136), which was detected in specimens from four locations. Genotypes Type IV, SCD-1, and SCD-2 were only found in one specimen, respectively $(0.7 \%, 1 / 136)$.

Similarly, four genotypes were identified (CD9, Type IV, $\mathrm{D}$, and PtEb IX) based on the analysis of the ITS sequences of the E. bieneusi-positive samples from stray cats (Table 2). Genotype CD9 was predominant, accounting for 50\% (11/ 22). This was followed by genotype Type IV $(27.3 \%, 6 / 22)$, which was detected in both Chengdu and Ya'an cities. Genotype D $(18.2 \%, 4 / 22)$ was detected in both Chengdu and Ya'an cities. Genotype PtEb IX was only found in one specimen in Chengdu city $(4.5 \%, 1 / 22)$.

\section{Phylogenetic analysis}

Phylogenetic analysis showed that all positive samples found in the present study belonged to two groups (group 1 and group 11). Genotypes D and Type IV were clustered into group 1 (Fig. 2). More importantly, the new genotype SCD-2 was also classified into the zoonotic group 1 (Fig. 2). Genotype CD9 and PtEb IX along with the new genotype SCD-1 were clustered into group 11 (Fig. 2). 


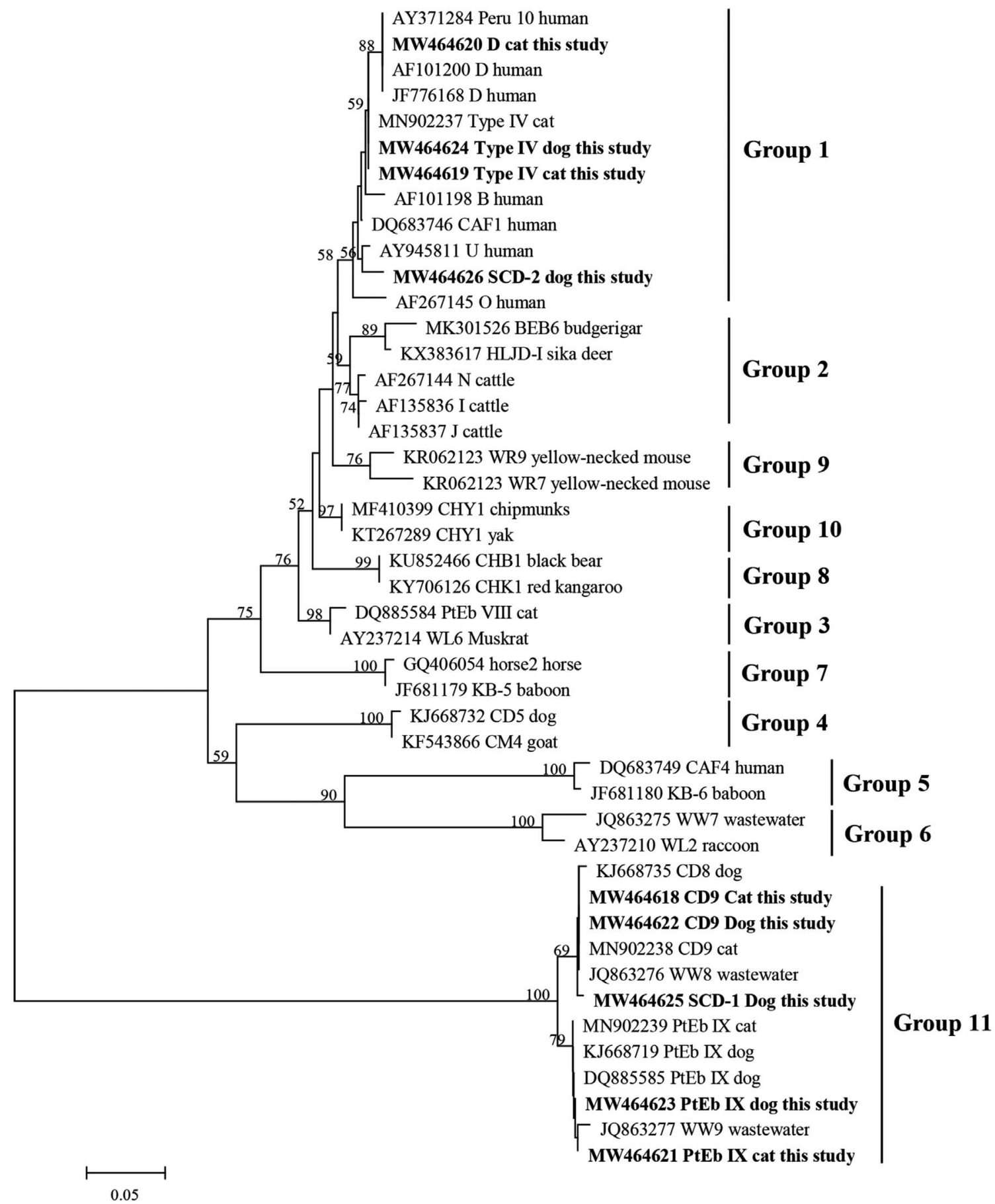

Figure 2. Phylogenetic relationship of Enterocytozoon bieneusi groups, the relationship between E. bieneusi genotypes obtained in the present study and other known genotypes deposited in GenBank was inferred by a neighbor-joining analysis of ITS sequences based on genetic distance by the Kimura-2-parameter model. The numbers on the branches represent percent bootstrapping values from 1000 replicates, with more than 50\% shown in the tree. Each sequence is identified by its accession number, genotype, and host. Genotypes obtained in this study are showed in bold.

\section{Discussion}

In the present study, the overall prevalence of E. bieneusi in sheltered dogs and cats was $18 \%$ (158/880). Specifically, we found the prevalence of E. bieneusi in stray dogs was $18.8 \%$ (136/724), which was higher than that $8.6 \%$ (27/315) in dogs in eastern China [16], and in Shanghai, China 6\% (29/485) [32]. In contrast, a more recent study in China revealed that the prevalence of E. bieneusi in dogs was 22.9\% (149/651) [29]. In cats, 22 cats (14.1\%) were found to be positive for E. bieneusi. The prevalence of E. bieneusi in cats in the present study was higher than that reported in previous studies in 
China, with ranges from $1.4 \%$ to $11.5 \%[10,16]$. The discrepancy in E. bieneusi prevalence may be associated with the fact that the fecal samples detected in the present study included those from sheltered dogs and cats, which lived in poor living conditions. Importantly, it has been determined that the poor living conditions were a major risk factor for contracting E. bieneusi infections [29].

In addition to China, E. bieneusi has been reported in dogs in various countries, with prevalence varying greatly. For example, the prevalence of E. bieneusi in stray dogs in Iran was the lowest, only $0.8 \%$ (2/237) [1]. Similarly, low E. bieneusi prevalence was also observed in dogs in Japan (4.4\%, 26/597) [19], and in Poland $(4.9 \%, 4 / 82)$ [20]. While high E. bieneusi prevalence was reported in a different study in Iran $(25.8 \%, 8 / 100)$ [8], up to $100 \%$ prevalence was recorded in Portugal and the United States [6, 17]. Several studies have determined that the prevalence of $E$. bieneusi in cats was also very different. Relatively low prevalence was found in Brazil $(3.3 \%, 2 / 60)$ [21], and in the Czech Republic (2.5\%, 3/118) [11]. In contrast, higher prevalence was reported in Colombia $(17.4 \%, 8 / 46)$ [26], and in Portugal $(100 \%, 6 / 6)$ [17]. However, we need to be cautious when interpreting this huge discrepancy, because many studies only included limited samples.

By sequence analysis of the ITS region of the rRNA of E. bieneusi, five genotypes were identified out of 136 E. bieneusi isolates from stray dogs, including three known and two novel genotypes (Table 1). Genotype CD9 showed predominance, accounting for $67.6 \%$ (92/136). Genotype CD9 was also reported in dogs and cats in southern China [29]. Genotype $\mathrm{PtEb}$ IX was the second most common in the present study, which was also the predominant genotype in dogs in various countries [10, 18, 19, 24]. Genotype Type IV has been identified in dogs in China and Colombia [10, 24]. Interestingly, two novel genotypes (SCD-1 and SCD-2) were identified for the first time in stray dogs, suggesting genetic variability of E. bieneusi to adapt to different host gut environments.

Similarly, four known genotypes were identified in stray cats in the present study. CD9 was the most common genotype in cats as well $(50 \%, 11 / 22)$. In a previous study, CD9 has been identified in household cats in Guangzhou province, southern China [29]. Type IV and D were the most common genotypes in cats in previous studies [10, 11, 16, 21, 32]. Genotypes D and Type IV are well known as zoonotic genotypes and have a broad-host range $[23,33]$. They have been reported in a wide range of animals, including non-human primates [9], chipmunks [2], captive wildlife [12], and domestic animals [13]. In this study, genotype PtEb IX was found in only one fecal sample, which is consistent with a previous study in cats in China [10]. However, genotype PtEb IX was the most common in cats in another study [29]. The discrepancy in prevalence of different genotypes may be related to geographical differences.

\section{Conclusion}

To the best of our knowledge, this is the first report of the prevalence and genotypes of E. bieneusi in sheltered dogs and cats in Sichuan province. The prevalence of E. bieneusi was $18.8 \%$ (136/724) and 14.1\% (22/156) in dogs and cats, respectively. Zoonotic genotypes D and Type IV have been identified in humans and were also reported in sheltered dogs and cats, implying that these animals infected with E. bieneusi may pose a potential threat to human health.

Acknowledgements. We would like to thank the National Science and Technology Department "13th five-year" Special Subproject of China (No. 2016YFD0501009). The funding had no role in designing the study, sample collection, analysis, and interpretation of data, or in writing the manuscript. Lei Deng was the recipient of scholarships from the Chinese Scholarship Council (CSC). We would also like to thank Wuyou Wang and Jingxin Yao for sample collection.

\section{References}

1. Dashti A, Santín M, Cano L, de Lucio A, Bailo B, de Mingo MH, Köster PC, Fernández-Basterra JA, Aramburu-Aguirre J, López-Molina N, Fernández-Crespo JC, Calero-Bernal R, Carmena D. 2019. Occurrence and genetic diversity of Enterocytozoon bieneusi (Microsporidia) in owned and sheltered dogs and cats in Northern Spain. Parasitology Research, 118, 29792987.

2. Deng L, Li W, Zhong Z, Chai Y, Yang L, Zheng H, Wang W, Fu H, He M, Huang X, Zuo Z, Wang Y, Cao S, Liu H, Ma X, Wu K, Peng G. 2018. Molecular characterization and new genotypes of Enterocytozoon bieneusi in pet chipmunks (Eutamias asiaticus) in Sichuan province. China. BMC Microbiology, 18(1), 37.

3. Deng L, Li W, Zhong Z, Gong C, Liu X, Huang X, Xiao L, Zhao R, Wang W, Feng F, Zhang Y, Hu Y, Fu H, He M, Zhang Y, Wu K, Peng G. 2016. Molecular characterization and multilocus genotypes of Enterocytozoon bieneusi among horses in southwestern China. Parasites \& Vectors, 9(1), 561.

4. Desportes I, Le Charpentier Y, Galian A, Bernard F, CochandPriollet B, Lavergne A, Ravisse P, Modigliani R. 1985. Occurrence of a new microsporidan: Enterocytozoon bieneusi n.g., n. sp., in the enterocytes of a human patient with AIDS. Journal of Protozoology, 32(2), 250-254.

5. Didier ES, Weiss LM. 2006. Microsporidiosis: current status. Current Opinion in Infectious Diseases, 19(5), 485-492.

6. Feng Y, Li N, Dearen T, Lobo ML, Matos O, Cama V, Xiao L. 2011. Development of a multilocus sequence typing tool for high-resolution genotyping of Enterocytozoon bieneusi. Applied and Environmental Microbiology, 77(14), 4822-4828.

7. Huang J, Zhang Z, Yang Y, Wang R, Zhao J, Jian F, Ning C, Zhang L. 2017. New Genotypes of Enterocytozoon bieneusi Isolated from Sika Deer and Red Deer in China. Frontiers in Microbiology, 8, 879.

8. Jamshidi Sh, Tabrizi AS, Bahrami M, Momtaz H. 2012. Microsporidia in household dogs and cats in Iran; a zoonotic concern. Veterinary Parasitology, 185(2-4), 121-123.

9. Karim MR, Dong H, Li T, Yu F, Li D, Zhang L, Li J, Wang R, Li S, Li X, Rume FI, Ning C. 2015. Predomination and new genotypes of Enterocytozoon bieneusi in captive nonhuman primates in zoos in China: high genetic diversity and zoonotic significance. PloS One, 10(2), e0117991.

10. Karim MR, Dong H, Yu F, Jian F, Zhang L, Wang R, Zhang S, Rume FI, Ning C, Xiao L. 2014. Genetic diversity in Enterocytozoon bieneusi isolates from dogs and cats in China: host specificity and public health implications. Journal of Clinical Microbiology, 52(9), 3297-3302.

11. Kvac M, Hofmannova L, Ortega Y, Holubova N, Horcickova M, Kicia M, Hlaskova L, Kvetonova D, Sak B, McEvoy J. 2017. Stray cats are more frequently infected with zoonotic protists than pet cats. Folia Parasitologica, 64(2017), 034. 
12. Li W, Deng L, Yu X, Zhong Z, Wang Q, Liu X, Niu L, Xie N, Deng J, Lei S, Wang L, Gong C, Zhou Z, Hu Y, Fu H, Xu H, Geng Y, Peng G. 2016. Multilocus genotypes and broad hostrange of Enterocytozoon bieneusi in captive wildlife at zoological gardens in China. Parasites \& Vectors, 9(1), 395.

13. Li W, Diao R, Yang J, Xiao L, Lu Y, Li Y, Song M. 2014. High diversity of human-pathogenic Enterocytozoon bieneusi genotypes in swine in northeast China. Parasitology Research, 113 (3), 1147-1153.

14. Li W, Feng Y, Santin M. 2019. Host specificity of Enterocytozoon bieneusi and public health implications. Trends in Parasitology, 35(6), 436-451.

15. Li W, Xiao L. 2020. Ecological and public health significance of Enterocytozoon bieneusi. One Health, 12, 100209.

16. Li WC, Qin J, Wang K, Gu YF. 2018. Genotypes of Enterocytozoon bieneusi in dogs and cats in Eastern China. Iranian Journal of Parasitology, 13(3), 457-465.

17. Lobo ML, Xiao L, Cama V, Stevens T, Antunes F, Matos O. 2006. Genotypes of Enterocytozoon bieneusi in mammals in Portugal. Journal of Eukaryotic Microbiology, 53(Suppl 1), S61-S64.

18. Mathis A, Breitenmoser AC, Deplazes P. 1999. Detection of new Enterocytozoon genotypes in faecal samples of farm dogs and a cat. Parasite, 6(2), 189-193.

19. Phrompraphai T, Itoh N, Iijima Y, Ito Y, Kimura Y. 2019. Molecular detection and genotyping of Enterocytozoon bieneusi in family pet dogs obtained from different routes in Japan. Parasitology International, 70, 86-88.

20. Piekarska J, Kicia M, Wesoõõlowska M, Kopacz Ż, Gorczykowski M, Szczepankiewicz B, Kváč M, Sak B. 2017. Zoonotic microsporidia in dogs and cats in Poland. Veterinary Parasitology, 246, 108-111.

21. Prado JBF, Ramos CADN, Fiuza VRDS, Terra VJB. 2019. Occurrence of zoonotic Enterocytozoon bieneusi in cats in Brazil. Brasileiro de Parasitologia Veterinaria, 28(1), 80-90.

22. Qi M, Li J, Zhao A, Cui Z, Wei Z, Jing B, Zhang L. 2018. Host specificity of Enterocytozoon bieneusi genotypes in Bactrian camels (Camelus bactrianus) in China. Parasites \& Vectors, 11 (1), 219.

23. Qiu L, Xia W, Li W, Ping J, Ding S, Liu H. 2019. The prevalence of microsporidia in China: A systematic review and meta-analysis. Scientific Reports, 9(1), 3174.

24. Santín M, Cortés Vecino JA, Fayer R. 2008. Enterocytozoon bieneusi genotypes in dogs in Bogota, Colombia. American Journal of Tropical Medicine and Hygiene, 79(2), 215-217.

25. Santín M, Fayer R. 2011. Microsporidiosis: Enterocytozoon bieneusi in domesticated and wild animals. Research in Veterinary Science, 90(3), 363-371.

26. Santín M, Trout JM, Vecino JA, Dubey JP, Fayer R. 2006. Cryptosporidium, Giardia and Enterocytozoon bieneusi in cats from Bogota (Colombia) and genotyping of isolates. Veterinary Parasitology, 141(3-4), 334-339.

27. Sulaiman IM, Fayer R, Lal AA, Trout JM, Schaefer FW 3rd, Xiao L. 2003. Molecular characterization of microsporidia indicates that wild mammals Harbor host-adapted Enterocytozoon spp. as well as human-pathogenic Enterocytozoon bieneusi. Applied and Environmental Microbiology, 69(8), 4495-4501.

28. Tian GR, Zhao GH, Du SZ, Hu XF, Wang HB, Zhang LX, Yu SK. 2015. First report of Enterocytozoon bieneusi from giant pandas (Ailuropoda melanoleuca) and red pandas (Ailurus fulgens) in China. Infection, Genetics and Evolution, 34, 32-35.

29. Wang H, Lin X, Sun Y, Qi N, Lv M, Xiao W, Chen Y, Xiang R, Sun M, Zhang L. 2020. Occurrence, risk factors and genotypes of Enterocytozoon bieneusi in dogs and cats in Guangzhou, southern China: high genotype diversity and zoonotic concern. BMC Veterinary Research, 16(1), 201.

30. Wang L, Zhang H, Zhao X, Zhang L, Zhang G, Guo M, Liu L, Feng Y, Xiao L. 2013. Zoonotic Cryptosporidium species and Enterocytozoon bieneusi genotypes in HIV-positive patients on antiretroviral therapy. Journal of Clinical Microbiology, 51(2), 557-563.

31. Wang Y, Zhang K, Zhang Y, Wang K, Gazizova A, Wang L, Cao L, Zhang Y, Huang J, Cui Y, Zhang Y, Zhang L. 2020. First detection of Enterocytozoon bieneusi in whooper swans (Cygnus cygnus) in China. Parasites \& Vectors, 13(1), 5.

32. Xu H, Jin Y, Wu W, Li P, Wang L, Li N, Feng Y, Xiao L. 2016. Genotypes of Cryptosporidium spp., Enterocytozoon bieneusi and Giardia duodenalis in dogs and cats in Shanghai, China. Parasites \& Vectors, 9, 121.

33. Yang Y, Lin Y, Li Q, Zhang S, Tao W, Wan Q, Jiang Y, Li W. 2015. Widespread presence of human-pathogenic Enterocytozoon bieneusi genotype $\mathrm{D}$ in farmed foxes (Vulpes vulpes) and raccoon dogs (Nyctereutes procyonoides) in China: first identification and zoonotic concern. Parasitology Research, 114(11), 4341-4348.

34. Zhang X, Wang Z, Su Y, Liang X, Sun X, Peng S, Lu H, Jiang N, Yin J, Xiang M, Chen Q. 2011. Identification and genotyping of Enterocytozoon bieneusi in China. Journal of Clinical Microbiology, 49(5), 2006-2008.

35. Zhang XX, Cong W, Lou ZL, Ma JG, Zheng WB, Yao QX, Zhao Q, Zhu XQ. 2016. Prevalence, risk factors and multilocus genotyping of Enterocytozoon bieneusi in farmed foxes (Vulpes lagopus), Northern China. Parasites \& Vectors, 9, 72.

36. Zhao W, Zhang W, Yang D, Zhang L, Wang R, Liu A. 2015. Prevalence of Enterocytozoon bieneusi and genetic diversity of ITS genotypes in sheep and goats in China. Infection, Genetics and Evolution, 32, 265-270.

Cite this article as: Zhong Y, Zhou Z, Deng L, Liu H, Zhong Z, Ma X, Zhang K, Wang Y, Fu H \& Peng G. 2021. Prevalence and new genotypes of Enterocytozoon bieneusi in sheltered dogs and cats in Sichuan province, southwestern China. Parasite $28,31$. 
An international open-access, peer-reviewed, online journal publishing high quality papers on all aspects of human and animal parasitology

Reviews, articles and short notes may be submitted. Fields include, but are not limited to: general, medical and veterinary parasitology; morphology, including ultrastructure; parasite systematics, including entomology, acarology, helminthology and protistology, and molecular analyses; molecular biology and biochemistry; immunology of parasitic diseases; host-parasite relationships; ecology and life history of parasites; epidemiology; therapeutics; new diagnostic tools.

All papers in Parasite are published in English. Manuscripts should have a broad interest and must not have been published or submitted elsewhere. No limit is imposed on the length of manuscripts.

Parasite (open-access) continues Parasite (print and online editions, 1994-2012) and Annales de Parasitologie Humaine et Comparée (1923-1993) and is the official journal of the Société Française de Parasitologie. 